\title{
Monitoreo de la transformación bainítica por medio de permeabilidad magnética ${ }^{\left({ }^{\circ}\right.}$
}

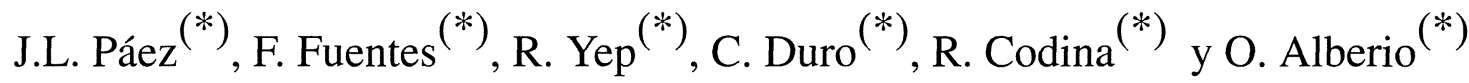

Resumen Se desarrolló un sistema que consta de una bomba axial de agitación del baño de sales de austempering y de un oscilador senoidal L-C de muy bajo nivel y amplitud controlada, cuya frecuencia depende de la inductancia formada por el conjunto bobina-probeta de ensayo. Los ensayos se realizaron entre 180 y $500{ }^{\circ} \mathrm{C}$. La transformación de la austenita de la probeta en el tiempo provoca variación en la inductancia de la bobina y, por lo tanto, la frecuencia de oscilación del sistema. Ésta se mide con un contador de cuatro dígitos cuya base de tiempos tiene la precisión aportada por un cristal de cuarzo piezoeléctrico. De esta forma, se logra monitorear la transformación simultáneamente con su desarrollo. Se construyó una interfase que permite leer los datos de la pantalla desde un ordenador para implementar una medición automática. Para contrastar el método, se ensayó el acero SAE 4140 con buenos resultados.

Palabras clave: ADI. Austempering. Diagrama TTT. Austenita retenida. Transformación bainítica.

\section{Bainitic transformation monitoring by a magnetic permeability method}

Abstract

\begin{abstract}
A system was developed which consists of an axial pump to stir the austempering liquid, and an L-C oscillator of a very low and checked amplitude level whose frequency depends on the coil test sample system. The test was performed at a temperature between 180 and $500{ }^{\circ} \mathrm{C}$. Along the time, the austenite transformation of the sample changes the coil inductance, and so the system oscillation frequency. The frequency is measured by a four digits counter that has a time base precision supplied by a piezoelectric quartz crystal. So, the transformation is monitored as it is going on. An interface was constructed in order to make possible data to be downloaded to a computer in order to have an automatic measurement. To contrast the method a SAE 4140 steel was tested and good results were achieved.
\end{abstract}

Keywords: ADI. Austempering. TTT Diagram. Retained austenite. Bainitic transformation.

\section{INTRODUCCIÓN}

El presente trabajo partió de la necesidad de monitorear la cinética de la transformación bainítica en la aleaciones férreas de alto contenido de silicio (fundición esferoidal -ADI- y aceros al silicio tipo SAE 92XX), debido a las peculiaridades que se producen en el desarrollo de la misma (1).

El objetivo fue el desarrollo de un sistema lo suficientemente sensible como para detectar las dos etapas de la transformación bainítica en estos materiales y que a la vez fuera lo bastante rápido como para disponer de la "ventana" del tratamiento en

(•) Trabajo recibido el día 4 de enero de 1995.

(*) Laboratorio de Metalografía y Laboratorio de Aplicaciones Experimentales. Facultad de Ingeniería. Universidad de Buenos Aires. Paseo Colón 850. Buenos Aires (Argentina). tiempos mucho más reducidos que el necesario con los métodos tradicionales.

Esta primera parte del trabajo trata sobre las características del sistema desarrollado y los resultados de las pruebas de contraste que se efectuaron con el acero SAE 4140.

\section{CARACTERÍSTICAS DEL SISTEMA DESARROLLADO}

\subsection{Conjunto bomba axial-bobina-probeta de ensayo}

El conjunto de esta bomba, diseñada especialmente para el crisol de sales de austempering disponible, está construido con acero inoxidable austenítico y recibe el movimiento de un motor eléctrico a través de una correa (Fig. 1). 


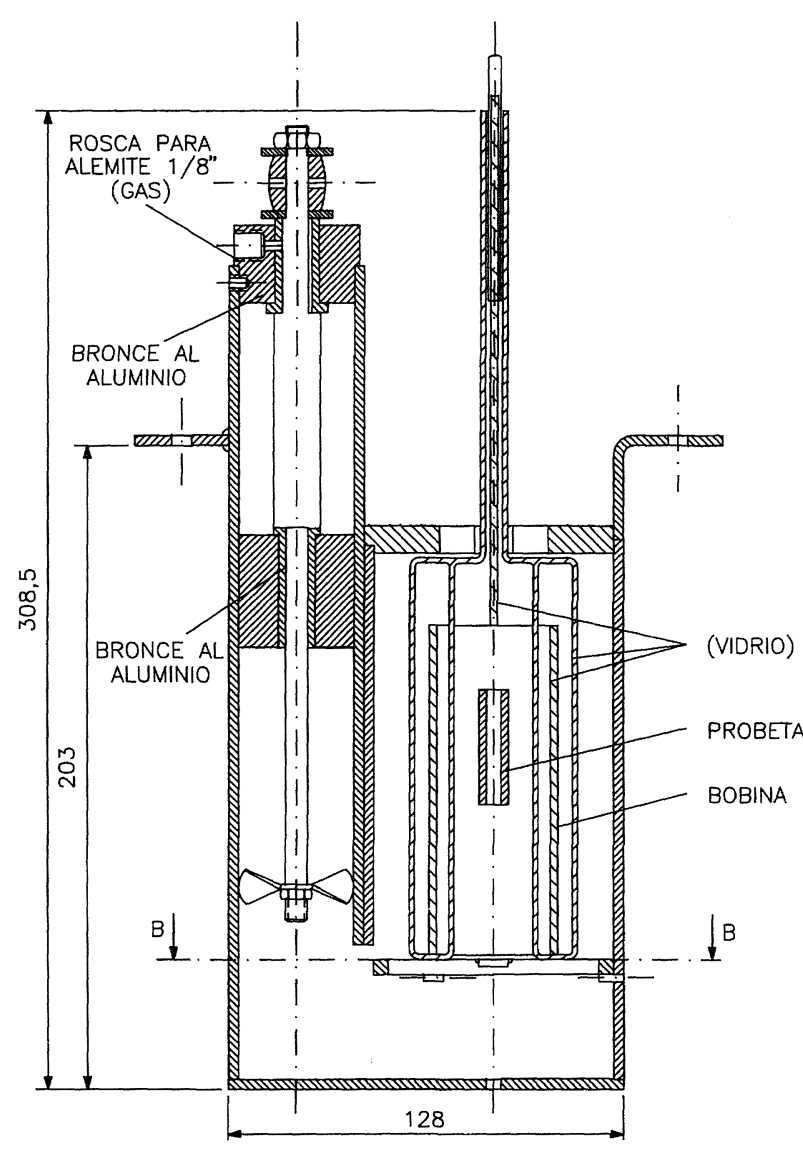

FIG. 1.- Corte longitudinal de la bomba axial, bobina y probeta de ensayo.

FIG. 1.-Axial pump, coil and test sample sectional view.

La probeta de ensayo es tubular y va montada en un soporte que permite su fácil manipulación desde la austenización hasta el horno de sales, a la vez que la posiciona en el centro de la bobina. Operativamente, el tiempo del traslado de la probeta desde el horno de austenización al de sales es de 2 a $4 \mathrm{~s}$.

La agitación del baño que provoca la bomba permite enfriar rápidamente la probeta desde la temperatura de austenización hasta la de austempering en tiempos que van desde 10 hasta $25 \mathrm{~s}$, según su masa.

\subsection{Oscilador senoidal L-C}

Se desarrolló y construyó un instrumento capaz de medir los cambios de la permeabilidad magnética de una probeta ferrosa en función del tiempo durante un tratamiento isotérmico en el rango 180$500{ }^{\circ} \mathrm{C}$.

Las características son las siguientes:

- La indicación del instrumento no necesita ser una función lineal de la permeabilidad, pero sí que sea monótona en el rango de medición. Esto se debe a que lo que se desea medir son las variaciones de las propiedades magnéticas de la probeta.

- Indicación digital sin necesidad de ajustes previos.

- Intervalos entre mediciones variables entre $2 \mathrm{~s} \mathrm{y}$ varios minutos.

- Equipo autosuficiente con interfase a ordenador para medición automática.

- Precisión y estabilidad superior al $1 \%$.

- Costo reducido.

Se decidió realizar la medición a través de un inductor cuyo núcleo es la probeta a ensayar. Consiste en un solenoide bobinado con alambre de cobre de $0,3 \mathrm{~mm}$ de diámetro con aislamiento de lana de vidrio y revestido en su totalidad por material no magnético ni conductor de electricidad (vidrio Pyrex) formado por un cilindro hueco en cuyo interior se coloca la probeta. Las condiciones geométricas son constantes, con lo que la inductancia es monótonamente creciente con la permeabilidad.

La inductancia de la bobina es de 2,4 mHy (en vacío, es decir, sin probeta en su interior) y su resistencia equivalente es variable con la temperatura hasta un máximo de $40 \Omega$ a $400{ }^{\circ} \mathrm{C}$. Por su simplicidad se adoptó una forma indirecta de medición a partir de la frecuencia de resonancia de un circuito L-C en paralelo.

$$
F_{r}=\frac{1}{2 \pi} \sqrt{\frac{1}{L \cdot C}-\frac{R^{2}}{L^{2}}}
$$

Considerando que $F r$ depende de la resistencia $R$ de la bobina, los valores se han elegido de modo tal que la función de $F r$ respecto a $L$ siempre tenga pendiente negativa; además, $R$ es un parámetro que no cambia durante la medición ya que la temperatura es constante.

El oscilador (a) (Fig. 2) consiste en el inductor con la probeta de ensayo y un capacitor muy estable con el tiempo y la temperatura. La oscilación se logra con dos transistores bipolares realimentados en forma positiva. La energía entregada al oscilador se controla eon una fuente de corriente continua (b). Como la amplitud es muy baja (del orden de $\mathrm{mV}$ ) se hace necesario amplificarla (c). El circuito (d) proporciona una tensión continua proporcional a la amplitud y controla la fuente (b) actuando como un control automático de ganancia. El frecuencímetro (f) se compone de un único circuito integrado que actúa sobre los cuatro dígitos de forma multiplexada. Posee una interfase paralela para registrar el dato con un ordenador. Las señales de control del frecuencímetro tienen una elevada estabilidad al ser generadas a partir de un cristal de cuarzo. 


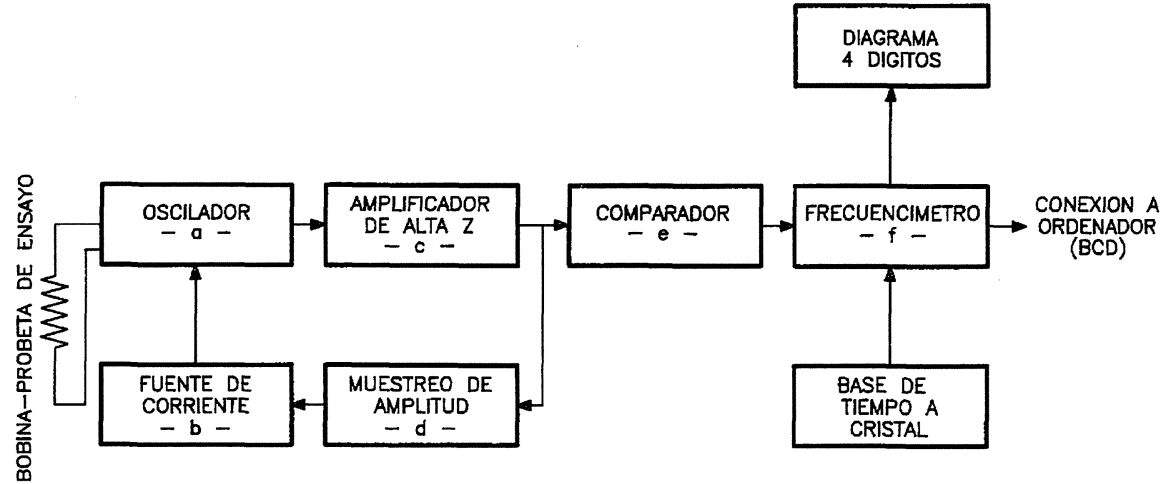

FIG. 2.-Diagrama de bloques del circuito electrónico.

FIG. 2.-Electronic circuit blocks diagram.

\subsection{Funcionamiento}

El avance de la transformación de la austenita hace variar la permeabilidad magnética del material $y$, por lo tanto, la frecuencia de oscilación del sistema. Los valores de frecuencia se presentan en un visor y además se introducen en un ordenador que los almacena en memoria durante el ensayo para su posterior análisis. Los cambios de los valores de frecuencia que se leen en pantalla son indicadores directos de la descomposición de la austenita.

Para contrastar el método, se ensayó el acero SAE 4140 y se compararon las características del diagrama TTT entre 250 y $440{ }^{\circ} \mathrm{C}$ obtenidas con el mismo, con las brindadas por las hojas de características.

\section{PROCEDIMIENTO EXPERIMENTAL}

Se realizaron tratamientos isotérmicos a 250 , $300,350,375,400$ y $440{ }^{\circ} \mathrm{C}$ con acero SAE 4140 . Se emplearon probetas de $90 \mathrm{~g}$ (de $20 \mathrm{~mm}$ de diámetro exterior) revestidas de pintura cerámica para evitar la descarburación durante la austenización, que se realizó a $840{ }^{\circ} \mathrm{C}$ durante $15 \mathrm{~min}$.

Se relevó la frecuencia absoluta en función del tiempo de austempering. Se consideró tiempo $0(t=$ $0)$ al momento en que la probeta quedó posicionada en el centro de la bobina dentro del baño de sales. Los resultados se muestran en la figura 3.

Los valores de frecuencia se almacenaron en el ordenador según los tiempos preprogramados.

\subsection{Condiciones térmicas del sistema y su influencia en la medición de frecuencia}

\subsubsection{Variaciones de temperatura en estado de equilibrio}

Una de las variables que influyen en la frecuencia es la resistencia de la bobina y por lo tanto la tempe-

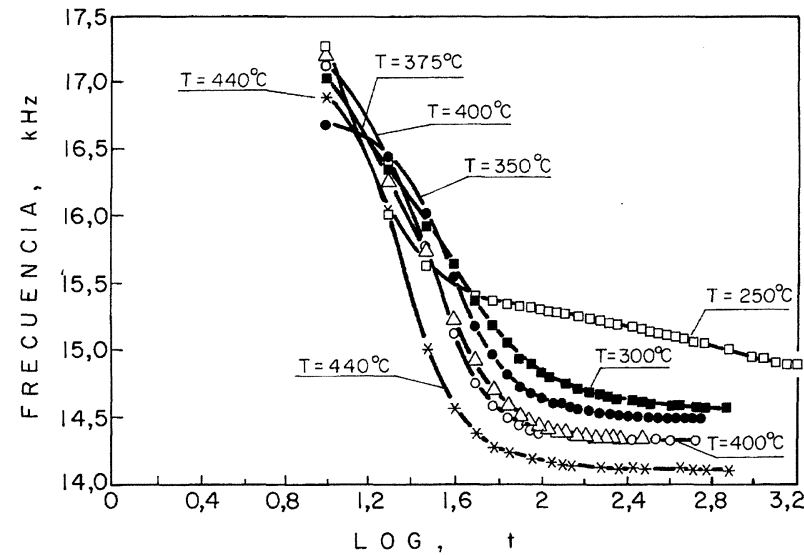

FIg. 3.- Frecuencia en función del tiempo de austempering.

FIG. 3.- Relationship between frecuency and austempering time.

ratura de tratamiento isotérmico. Se determinó una relación lineal (con coeficiente de correlación 0,99) entre frecuencia y temperatura del baño de sales con respecto a microestructura constante en el rango 250$350{ }^{\circ} \mathrm{C}$ (Fig. 4). La pendiente es de $3,8 \mathrm{~Hz} /{ }^{\circ} \mathrm{C}$.

$\mathrm{Si}$ bien el ensayo se realizó a temperatura constante, hubo desvíos con respecto a la nominal de

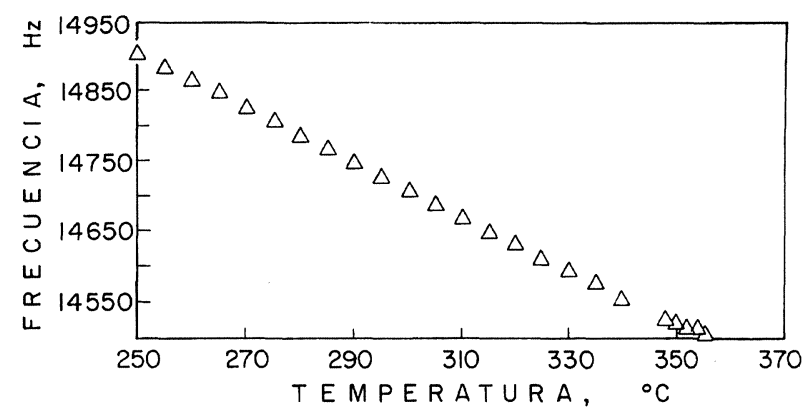

FIG. 4.- Frecuencia en función de la temperatura de austempering.

FIG. 4.- Relationship between frecuency and austempering temperature. 
hasta $3{ }^{\circ} \mathrm{C}$. En estos casos, se corrigió el valor de frecuencia medida según la relación encontrada de $3,8 \mathrm{~Hz} /{ }^{\circ} \mathrm{C}$. Esto fue posible, pues, conjuntamente con el valor de frecuencia, se registró la temperatura del baño en cada medición.

En la figura 3 se representan gráficamente los valores de frecuencia corregidos.

\subsubsection{Enfriamiento inicial desde la temperatura de austenización}

Hay un período transitorio térmico en los primeros momentos en los que la temperatura de la probeta pasa de $840{ }^{\circ} \mathrm{C}$ hasta la del baño de sales: la duración de este período es del orden de $25 \mathrm{~s}$.

La velocidad de enfriamiento de la probeta es lo suficientemente rápida como para evitar la "nariz" de perlita del acero SAE 4140, pero los valores de frecuencia registrados no pueden corregirse como se indicó en el párrafo anterior, pues la relación 3,8 $\mathrm{Hz} /{ }^{\circ} \mathrm{C}$ deja de ser válida. Por este motivo, en las figuras 3 y 5 las curvas no arrancan de tiempo 0 .

\subsection{Relación frecuencia-porcentaje de transformación de austenita}

La frecuencia no se utiliza como valor absoluto. Se refiere a la correspondiente al $0 \%$ de transformación, es decir, al material en estado completamente austenítico.

La relación entre variación de frecuencia y el porcentaje de transformación es del tipo

$$
\Delta F r=A\left(1-e^{(-\mathrm{aT})}\right)
$$

donde

$\Delta F r=$ Variación de frecuencia con respecto al estado austenítico ( $0 \%$ de transformación).

$T=$ Porcentaje de transformación de la austenita . $A, a=$ Constantes, dependen de la temperatura.

Para determinar $A$ y $a$ se trataron isotérmicamente probetas de distintas masas $(45,56,65,78 \mathrm{y}$ $90 \mathrm{~g}$ ) a $350{ }^{\circ} \mathrm{C}$. Todas las probetas eran iguales geométricamente excepto en el diámetro exterior. De estos ensayos se registró la frecuencia para el 100 $\%$ de transformación en cada probeta a las temperaturas de $250,300,350,375,400$ y $440{ }^{\circ} \mathrm{C}$. Se consideró la variación total de frecuencia de cada probeta como equivalente a la variación de $90 \mathrm{~g}$ pero con sólo una fracción de austenita transformada. Por ejemplo la $\Delta F r(T=100 \%)$ de la probeta de 45 g se consideró como equivalente a la $\Delta F r$ de la probeta de 90 g cuando $T=50 \%$.
No se pudieron medir las frecuencias para las probetas en estado completamente austenítico $(0 \%$ de transformación) por lo mencionado anteriormente y porque el inicio de la transformación bainítica se produce a $6 \mathrm{~s}$ en el acero SAE 4140 a $350{ }^{\circ} \mathrm{C}$. A partir de entonces, se prepararon probetas de acero inoxidable austenítico de distintas masas que representaron dicho estado: así se registró la frecuencia de referencia a cada temperatura.

Por el método de los mínimos cuadrados se calcularon las constantes $A$ y $a$ para cada temperatura obteniéndose un muy buen coeficiente de correlación $\left(r^{2}=0,96\right)$.

Con la relación entre $\Delta F r$ y el porcentaje de transformación de la austenita, se obtuvieron las curvas de transformación en función del tiempo que muestra la figura 5 .

\section{ANÁLISIS DE LOS RESULTADOS OBTENIDOS}

Para determinar la relación entre el porcentaje de transformación de la austenita con la variación de frecuencia se estableció la hipótesis de que la microestructura no influye en la variación de frecuencia total para igual masa de probeta, y la que influye es la temperatura. Esta hipótesis ya se utilizó con éxito en un trabajo similar (2).

Para $350,375,400$ y $440{ }^{\circ} \mathrm{C}$, los puntos del $50 \mathrm{y}$ $100 \%$ de la transformación se corresponden con los diagramas TTT del acero 4140 que ofrecen los manuales (Fig. 6)(3).

Dada la masa de la probeta de ensayo (90 g), no se pudo llegar al equilibrio térmico a tiempo suficiente como para detectar el inicio de la transformación, que para el acero SAE 4140 está entre los 3 y $6 \mathrm{~s}$ en el campo bainítico.

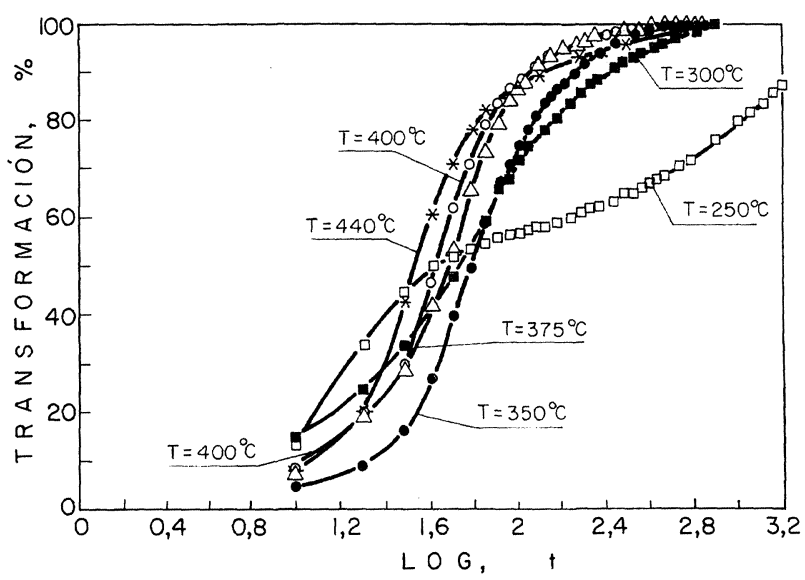

FIG. 5.- Porcentaje de transformación de la austenita en función del tiempo de austempering.

FIG. 5.- Relationship between austenite transformation percentage and austempering time. 


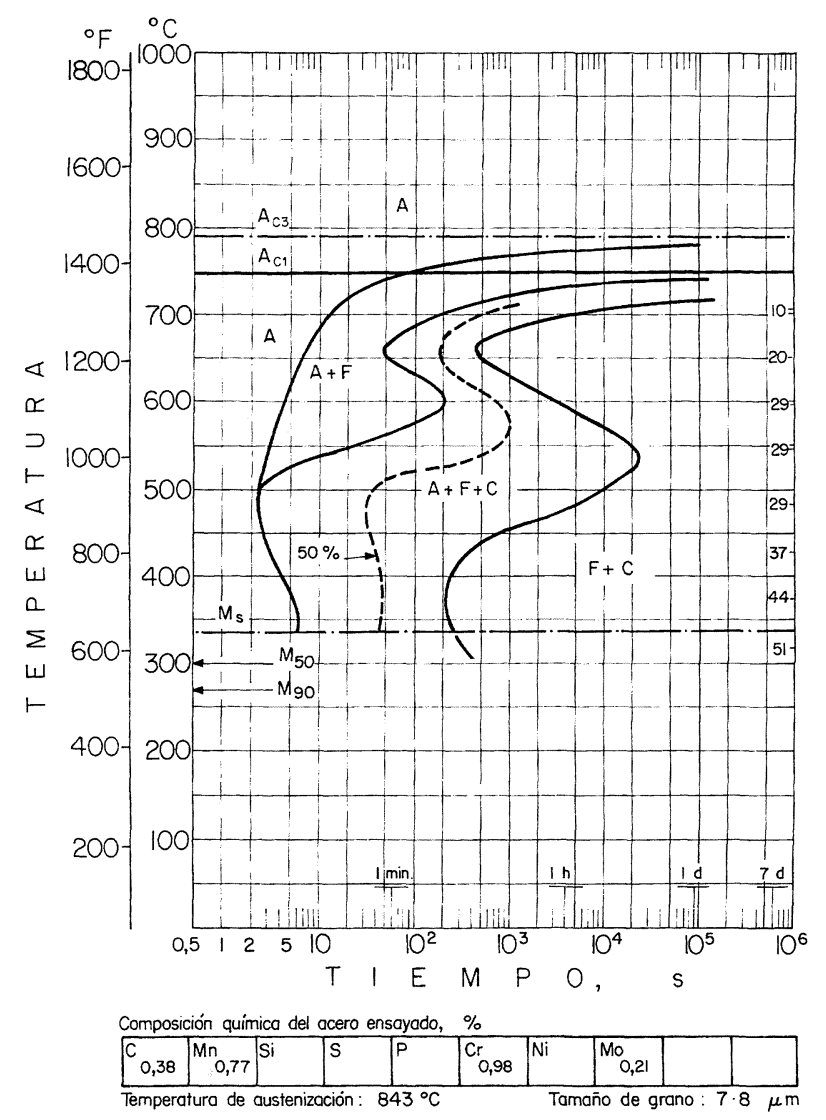

FIG. 6.- Diagrama de transformación isotérmica del acero SAE 4140.

FIG. 6.-TTT diagram of the SAE 4140 steel.

A 300 y $250{ }^{\circ} \mathrm{C}$ se detectó la transformación martensítica seguida por la transformación de la austenita no transformada. Sin embargo, no se pudo determinar con precisión el porcentaje de transformación de martensita debido al mismo problema mencionado en el apartado 3.2., aunque se percibe que corresponden valores menores que los indicados en el diagrama TTT de este material.

El método es lo suficientemente sensible como para detectar variaciones del $0,5 \%$. La incertidumbre en el cálculo del valor porcentual de transformación de austenita es menor del $1 \%$.
Mecánicamente, el sistema se comportó sin presentar inconvenientes, tanto en lo que se refiere al funcionamiento de la bomba axial como a la integridad de la bobina. Esto es particularmente significativo dada la alta temperatura a la que están directamente sometidos estos elementos.

\section{CONCLUSIONES}

Se logró desarrollar un método de monitoreo de transformación de la austenita que es muy adecuado para ADI y ASIS por ser muy sensible a la descomposición de esta fase.

Es un método directo pues se aplica in situ, mientras la transformación se está desarrollando, con lo cual la determinación de la "ventana" para ADI y ASIS será muy rápida.

Se pueden determinar porcentajes de austenita retenida, pues se encontró la relación entre este valor y el parámetro que se mide (frecuencia de oscilación del circuito L-C).

. El método se puede generalizar para la caracterización de cualquier aleación férrica bainítica y martensítica con tal de efectuar algunos ajustes en las condiciones térmicas de enfriamiento de la probeta siempre que se alcancen las temperaturas de tratamiento isotérmico dentro de los $10 \mathrm{~s}$.

\section{Agradecimiento}

Los autores agradecen a la Escuela Superior Técnica del Ejército la realización de los ensayos correspondientes.

\section{REFERENCIAS}

(1) Le Houllier, R., BÉGin, G. y Dubé, A. Metall. Trans., 2 (9), 1971: 2.645-2.653

(2) BABU, B.N.P., Bhat, M.S., PARKer, E.R. y ZaCKAy, V.F. Metall. Trans. A, 7A (1), 1976: 17-22.

(3) IRAM-IAS. Acero para construcciones mecánicas-Hojas de características. 